\title{
SLR Contribution to Investigation of Polar Motion
}

\author{
Zinovy Malkin \\ Institute of Applied Astronomy \\ nab. Kutuzova 10, St.Petersburg 191187, Russia
}

\begin{abstract}
The Satellite Laser Ranging (SLR) technique has been used to determine Earth Rotation Parameters (ERP) for over twenty years. Most of results contributed to the International Earth Rotation Service (IERS) are based on analysis of observations of Lageos 1 \& 2 satellites collected by the global tracking network of about 40 stations. Now five analysis centers submit operational (with 2-15 days delay) solutions and about ten analysis centers contribute yearly final (up to 23 years) ERP series. Some statistics related to SLR observations and analysis are presented and analyzed. Possible problems in SLR observations and analysis and ways of its solution are discussed.
\end{abstract}

\section{Introduction}

Laser ranging to artificial satellites was initiated in 1964 after the launch of the first geodetic satellite Beacon-B. Since that time satellite laser ranging (SLR) has been widely used for geodynamical and geophysical research. The primary fields of investigations using SLR observations are Earth rotation, maintenance of the Terrestrial Reference Frame, tectonic motion, crustal deformations, geopotential with its spatial and temporal variations, tides, movement of the geocenter, support of satellite geophysical missions (such as satellite altimetry), global time transfer, and others. Detailed analysis of SLR contributions to Earth sciences can be found in (Tapley et al. 1993).

After the launching of the Lageos satellite in May 1976, SLR became one of the main techniques to investigate Earth rotation, and for over twenty years, the SLR technique has been used to determine ERP. Most of the results contributed to the IERS are based on analysis of observations of Lageos $1 \& 2$ satellites collected by the global tracking network of about 40 stations. SLR provides high precision series of $\mathrm{x}, \mathrm{y}$, and LOD. Some analysis centers compute also UT to densify Universal Time series in combination with VLBI data.

The importance of SLR as one of the main methods to study the Earth led, naturally, to the establishment of the International Laser Ranging Service (ILRS) in 1998 to coordinate scientific activity SLR (and LLR), chiefly in the framework of IAG and IERS projects.

In this paper we will focus only on the SLR contribution to the investigation of polar motion in accordance with the topics of the conference. 
Combined

Compared

\begin{tabular}{|c|c|c|c|c|c|c|c|c|c|c|c|c|c|c|c|c|c|c|c|}
\hline Center 1 & 79 & 80 & 81 & 82 & 83 & 84 & 85 & 86 & 87 & 88 & 89 & 90 & 91 & 92 & 93 & 94 & 95 & 96 & 97 \\
\hline \multicolumn{20}{|c|}{ BKG/IFAG, DE } \\
\hline \multirow{2}{*}{\multicolumn{20}{|c|}{$\begin{array}{l}\text { CGS, IT } \\
\text { CLG, BU } \\
\end{array}$}} \\
\hline & & & & & & & & & & & & & & & & & & & \\
\hline \multicolumn{20}{|c|}{ CSR/LASOM, US } \\
\hline \multicolumn{20}{|c|}{ DGFI, DE } \\
\hline \multicolumn{20}{|l|}{ DUT, NL } \\
\hline \multirow{2}{*}{\multicolumn{20}{|c|}{\begin{tabular}{|l|} 
ESOC, DE \\
GAOUA, UA \\
\end{tabular}}} \\
\hline & & & & & & & & & & & & & & & & & & & \\
\hline \multicolumn{20}{|c|}{ GFZ/ZIPE, DE } \\
\hline \multicolumn{20}{|c|}{ GRGS, FR } \\
\hline \multicolumn{20}{|c|}{ GSFC, US } \\
\hline \multicolumn{20}{|l|}{ GZ, RU } \\
\hline \multicolumn{20}{|l|}{$\mathbf{I A A}, \mathbf{R U}$} \\
\hline \multicolumn{20}{|c|}{ IARAS, RU } \\
\hline \multicolumn{20}{|c|}{ IMVP, RU } \\
\hline \multicolumn{20}{|l|}{$\mathbf{N A L}, \mathbf{J P}$} \\
\hline \multicolumn{20}{|l|}{ SAO, US } \\
\hline SHA, CN & & & & & & & & & & & & & & & & & & & \\
\hline
\end{tabular}

Figure 1. Contribution of SLR ACs to IERS yearly solutions.

\section{Contribution of SLR PM series to the IERS}

SLR observations of Lageos and ERP derived from these observations are available from May, 1976. However, only in 1980-81 after significant improvement of ranging techniques did the SLR ERP series achieve the accuracy required for investigation of polar motion (PM). Figure 1 summarizes information from the IERS Annual Reports for 1978-1997 concerning the use of submitted SLR series in IERS yearly solutions. One can see that beginning from 1983, the SLR ERP series is regularly used for IERS combination. It's also seen that the Center for Space Research, University of Texas at Austin (CSR) provides the longest stable PM series.

At present five analysis centers submit operational solutions with 2-15 days delay, and about ten analysis centers contribute yearly final (up to 23 years) ERP series. The longest series available for analysis are listed in Table 1.

\section{Accuracy of SLR PM series}

During the long history of using the SLR technique to study, PM drastic improvement in range precision was achieved. It's interesting to see how this technology development affected the accuracy of the PM series obtained from SLR observations. Figure 2 shows differences between SLR series obtained at the CSR and the Institute of Applied Astronomy, St.Petersburg (IAA) and IERS combined solution EOP(IERS)C04.

One can clearly see in Figure 2 that replacement of the first generation by the second generation ranging equipment about 1980 and its further replacement by the third generation units led to significant improvement of the accuracy of 
Table 1. Longest series.

$\begin{array}{ll}\text { CSR } & 1976- \\ \text { GSFC } & 1980- \\ \text { DUT } & 1983- \\ \text { GAOUA } & 1983- \\ \text { IAA } & 1983= \\ \text { CGS } & 1985- \\ \text { BKG } & 1988-\end{array}$

Table 2. Agreement of series with $\mathrm{C} 04$, mas

Solution $\quad X_{p} \quad Y_{p}$

$\begin{array}{lll}\text { GPS } & 0.11 & 0.11 \\ \text { SLR } & 0.11 & 0.12 \\ \text { VLBI } & 0.16 & 0.15\end{array}$

Table 3. Statistics of operational solutions based on USNO data for Sep 1998 - Sep 1999.

Center Delay, days Error in PM mas

\begin{tabular}{lrrr}
\hline & & & \\
NEOS VLBI & 6.3 & 12.0 & 0.10 \\
IAA VLBI & 13.3 & 22.5 & 0.12
\end{tabular}

$\begin{array}{lrrr}\text { CSR SLR } & 2.3 & 9.2 & 0.36 \\ \text { TUD SLR } & 4.6 & 7.8 & 0.36 \\ \text { IAA SLR } & 1.6 & 3.1 & 0.20 \\ \text { MCC SLR } & 7.6 & 11.1 & 0.21 \\ & & & \\ \text { IGS Final } & 10.1 & 14.8 & 0.02 \\ \text { IGS Rapid } & 1.1 & 1.4 & 0.06\end{array}$
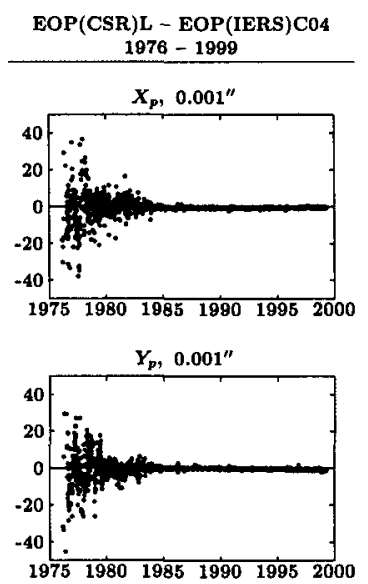
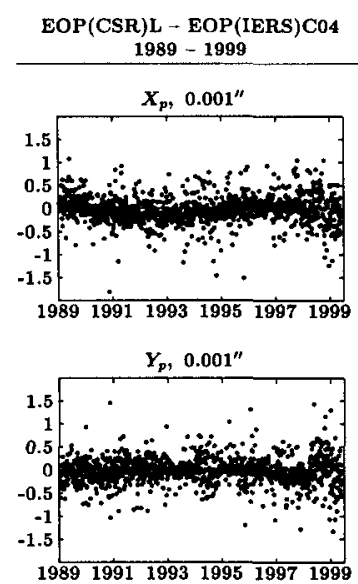
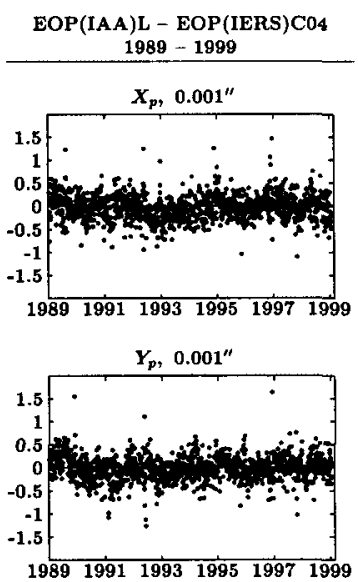

Figure 2. Differences between SLR and IERS PM series. 
the PM solution. However, in spite of the improvement of ranging technique and implementation of the fourth generation equipment, the precision of PM series derived from SLR observations remains the same during last 10-12 years. We will discuss the problem below.

In 1998 the IERS Central Bureau derived the final IERS combination in two steps. In the first stage combined VLBI, SLR, and GPS series was computed and then this was used for final IERS combination. Table 2 from the 1997 IERS Annual Report shows that the precision of SLR and GPS combinations is practically the same. Unfortunately, this is not so for operational solutions.

Table 3 compiled on basis of gpspol. asc files produced by USNO along with Bulletin A issues contains statistics of series used for the IERS Rapid Service. Of course, errors in PM obtained there depend on weighting applied to combined series, but it already clear that the accuracy of individual SLR series is worse than VLBI and GPS ones, and the delay of SLR contribution is much worse than for GPS. Nevertheless, one can see that at least two centers can produce operational solutions with delays of about 2 days. If all SLR analysis centers would provide such a solution on a regular basis, it will allow us to have, in principle, a combined SLR solution with accuracy $0.1-0.15$ mas and delay $1.5-2$ days.

\section{Factors limited quality of the SLR ERP solutions}

For a long time SLR was the one of the main methods to determine PM and to densify the PM series based on VLBI observations. However, lately SLR ERP series are inferior to GPS in quality (accuracy, density, delay of operational solution) of results. It would be very important to understand existing problems in SLR observations and analysis procedures and to discuss possible ways to solve them.

\subsection{Observations}

The first problem with SLR observations is that SLR is a 'one-object' technique. This means that, unlike GPS, SLR stations can observe only one object at a time. Hence, planning of observations and priority politics plays a substantial role in acquisition of observations of satellites on which PM determination is based, especially keeping in mind that SLR observations are rather expensive and that the number of ranging units is limited.

At present four operational satellites seem most suitable to investigate Earth rotation (as well as tectonic motion and long-term temporal variations in various geophysical parameters) - two Lageos satellites and two Etalon satellites. Both Lageos and Etalon satellites were launched into long-time stable orbits and have a low area-to-mass ratio. Their descriptions are presented in Figure 3.

Figure 4 shows the number of observations of these satellites during the last 12 years and ILRS priorities. One can see that the number of observations of Lageos satellites remains approximately the same during these years and that the number of observations of Etalon satellite is too small to contribute seriously to analysis.

Figure 5 shows the distribution of observations by stations for the period from Sep 1983 to Aug 1999. Table 4 contains a list of stations that contributed 


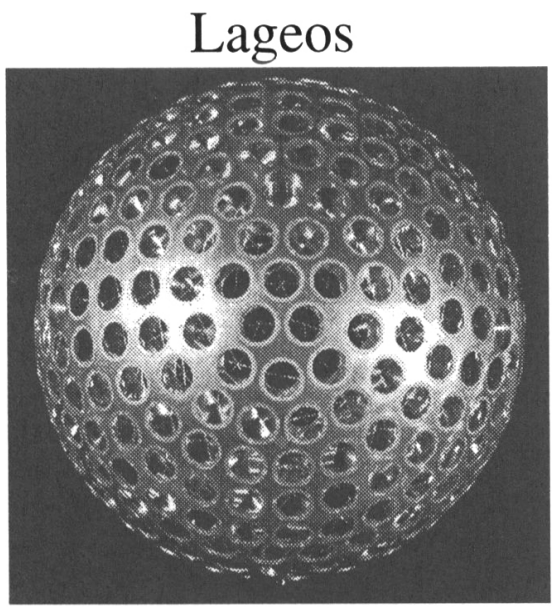

\section{$\underline{\text { Satellite }}$

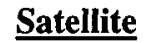

Lageos 1,2 Etalon-1,2

\section{Satellite parameters} $D, m \quad M, k g \quad A / M, m^{\wedge} 2 / k g$

0.6 407

0.00070

0.00098

\section{Etalon}

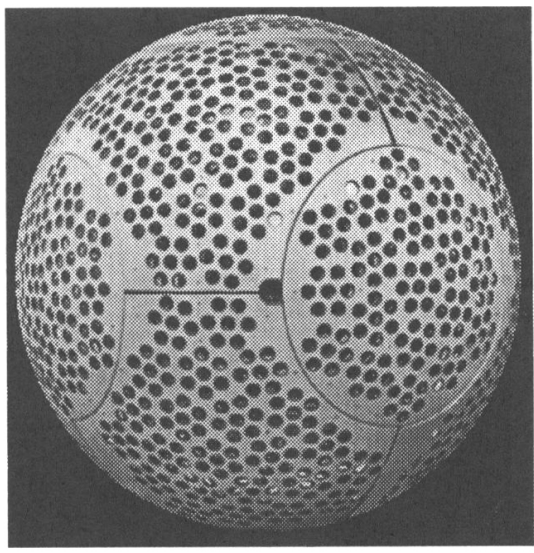

\section{Orbital parameters}

a, km h, km T, h i, deg $12300 \quad 5900 \quad 3.76 \quad 110,53$ $\begin{array}{llll}25500 & 19100 & 11.26 & 65\end{array}$

Figure 3. Satellites Lageos and Etalon.

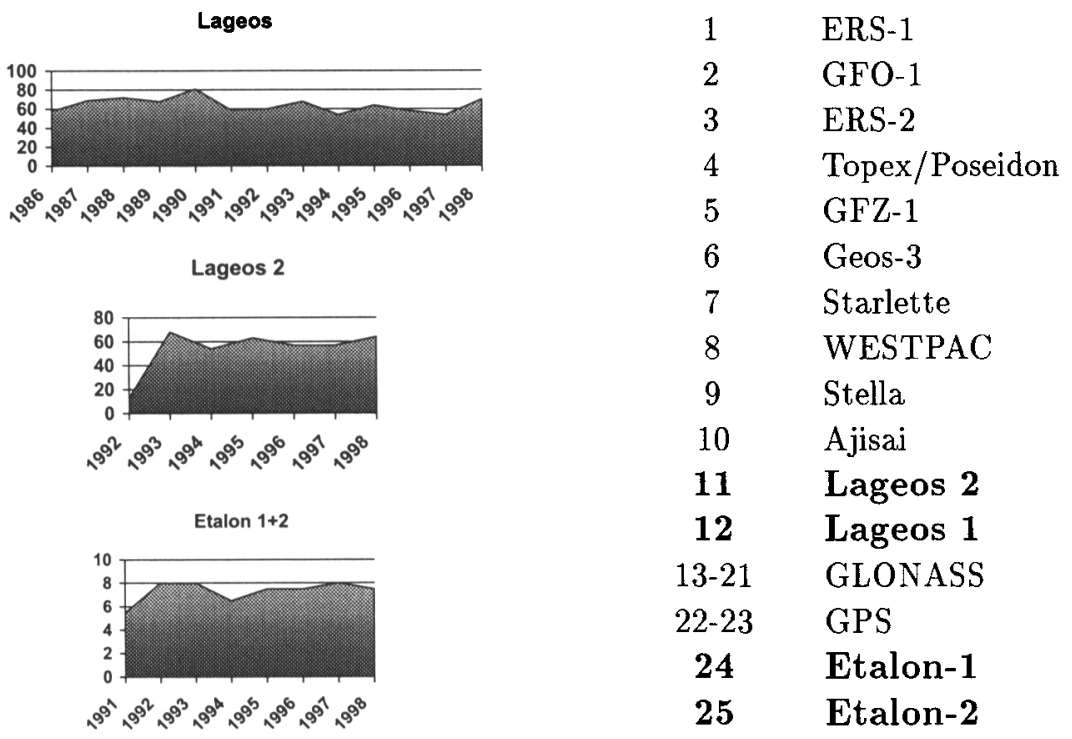

Figure 4. Number of observations (normal points), thousands (at the left) and ILRS priorities (at the right) of satellites. 


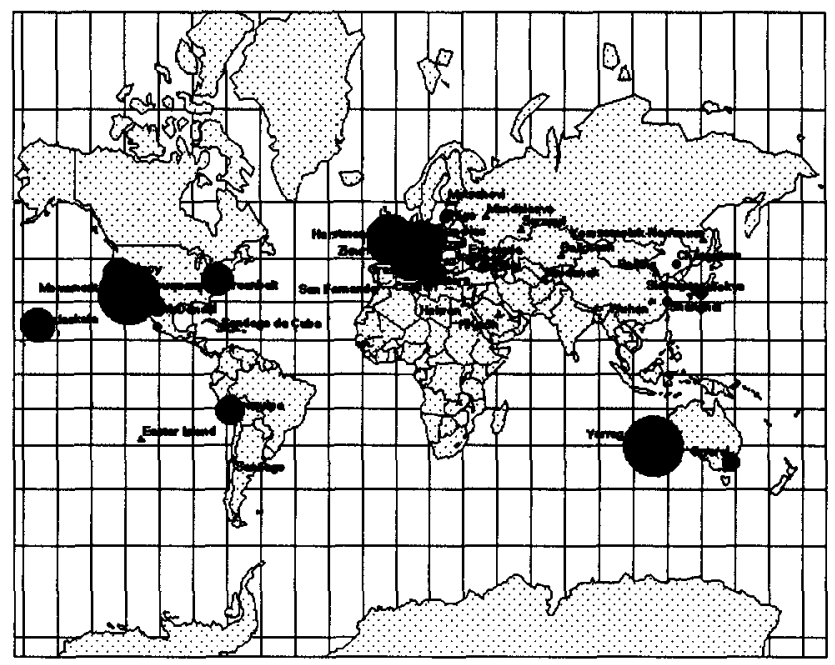

Figure 5. Distribution of observations in stations.

more than $2 \%$ of the total number of observations for the whole period and during the last year (in parenthesis).

The first impression is that stations are distributed quite uniformly along the satellite orbit. However, if we recall that SLR observations are weatherdependent, it would be evident that the Australia - West Asia region need at least one more active station. Fortunately, several new stations were put into operation in China and Japan during the last few years, but they are still not active enough. So, the problem of more uniform distribution of observations by station evidently still exists. Besides, as usual, most of the stations are located in the northern hemisphere and at least two active stations would be very desirable in the southern hemisphere (in South America and South Africa).

Distribution of observations in months is more or less uniform, with a small decrease in November and December (sharp dip during Christmas days!). Distribution by days of week (Figure 6) is not so good. One can see that number of observations for several of the most active stations is about half that during workdays. These are the statistics for the whole interval from Sep 1983 to Aug 1999. Last year's distribution is a little bit more uniform - the maximum number of observations was made on Wednesday (16.2\%), and the minimum on Sunday $(11.6 \%)$, but still far from ideal.

\subsection{Analysis}

Though, in my opinion, the only real way to improve the quality of SLR ERP series is to increase the number of observations and, especially, the number of satellites involved in the determination of ERP, the analysis strategy can also be improved. 
Table 4. Distribution of observations in stations

\begin{tabular}{llcclc} 
CDP & \multicolumn{1}{c}{ Station } & \multicolumn{1}{c}{$\%$} & CDP & \multicolumn{1}{c}{ Station } & $\%$ \\
\hline 7110 & MONUMENT PEAK & $9.6(12.6)$ & 7843 & CANBERRA & $3.0(0.3)$ \\
7090 & YARRAGADEE & $9.3(10.0)$ & 7403 & AREQUIPA & $2.7(1.2)$ \\
7840 & HERSTMONCEUX & $8.2(8.7)$ & 7810 & ZIMMERWALD & $2.4(3.6)$ \\
7210 & MAUI & $5.4(1.6)$ & 7838 & SIMOSATO & $2.4(0.8)$ \\
7835 & GRASSE & $5.3(2.9)$ & 7907 & AREQUIPA & $2.0(-)$ \\
7105 & WASHINGTON & $5.3(6.3)$ & 7836 & POTSDAM & $1.7(2.1)$ \\
7839 & GRAZ & $5.0(7.9)$ & 1884 & RIGA & $1.5(2.2)$ \\
7109 & QUINCY & $4.8(-)$ & 7237 & CHANGCHUN & $1.2(2.5)$ \\
7939 & MATERA & $4.6(3.7)$ & 7811 & BOROWIEC & $1.0(2.3)$ \\
8834 & WETTZELL & $4.0(4.8)$ & 7849 & MOUNT STROMLO & $0.8(7.9)$ \\
7080 & FORT DAVIS & $3.3(4.0)$ & 7845 & GRASSE & $0.6(2.7)$
\end{tabular}

Total about 130 stations including mobile occupations

5 stations provided $38 \%$ of total number of observations

7 stations provided $\mathbf{4 8 \%}$ of total number of observations

10 stations provided $62 \%$ of total number of observations

15 stations provided $75 \%$ of total number of observations

22 stations provided $\mathbf{8 5 \%}$ of total number of observations

The modern tendency is to use combined VLBI, GPS, SLR series for the final IERS combination. Unfortunately, PM series produced by SLR analysis centers are not unified enough, which makes their comparison and combination difficult. The main problems in individual SLR series are:

- Sparse time series (3-5 days for most solutions, 1 day needed).

- Large delay of operational solutions (5-10 days for most solutions, 1-2 days needed).

- Non-unified Terrestrial Reference System (TRS).

- Dependence on a priori values of EOP.

Analysis of methods used to determine ERP from SLR observations shows that not all possibilities for improvement and unification of SLR ERP series are exhausted. What can we do to make SLR series more efficient? Existing experience shows that significant improvement could be achieved if we would:

- Produce daily EOP series.

- Produce operational solutions with delays of about 2 days.

- Improve the strategy to determine UT.

- Use the same TRS for all solutions. The International Terrestrial Reference Frame (ITRF) seems most suitable for this purpose. 
20

15

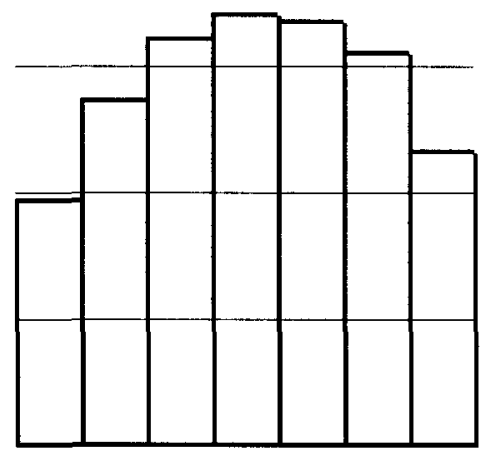

Sun Mon Tue Wed Thu Fri Sat

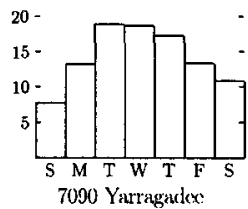

$20-$
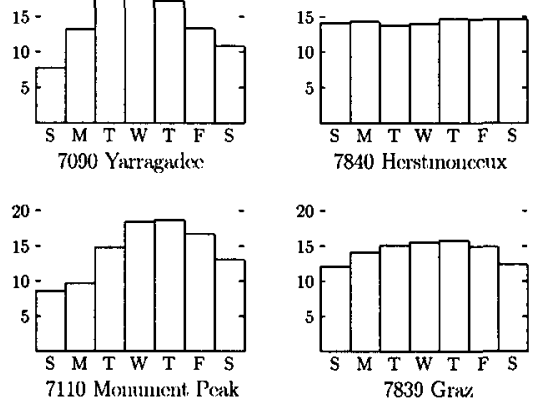

20 -
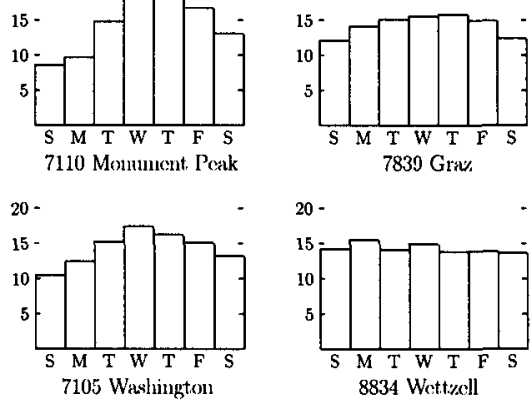

Figure 6. Distribution of observations in days of week.

An analysis of these problems and some proposals for their solution was presented in (Malkin 1998). Below proposed methods tested by the IAA during the last three years are briefly described.

The first serious problem is how to compute daily ERP series without loss of accuracy. There are two main ways to compute daily series. The first is to use daily arc solutions (i.e. use one-day arcs). But this leads to degradation of the accuracy of the solution. The second is to use overlapping arcs with appropriate length and a one-day shift between arcs. Such a solution produces correlated results for neighboring arcs, but significant improvement in precision can be considered as sufficient compensation. Table 5 shows accuracy of ERP solution with various arc lengths. Experience shows that using overlapping arcs yields more precise ERP series than using one-day arcs.

Table 5. Dependence of errors in ERP on arc length (RMS of EOP(IAA)L - EOP(IERS)C04 after removing trend).

\begin{tabular}{lccccc}
\multirow{2}{*}{ ERP } & \multicolumn{5}{c}{ Length of arc, days } \\
\cline { 2 - 6 } & 1 & 3 & 4 & 5 & 7 \\
\hline$X, 0.001^{\prime \prime}$ & 0.40 & 0.23 & 0.20 & 0.18 & 0.16 \\
$Y, 0.001^{\prime \prime}$ & 0.34 & 0.22 & 0.21 & 0.18 & 0.15 \\
$L O D, 0.0001 s$ & 0.44 & 0.16 & 0.15 & 0.12 & 0.09
\end{tabular}

It would be very desirable to have strictly daily series for all analysis centers, i.e. series with epochs equal to $0^{h}$. This can be achieved by two methods determination of pole coordinates with their rates and a posteriori interpolation 
to $0^{h}$. Numerous test showed that including pole coordinates rates into the parameter model led to small degradation of accuracy of PM, evidently due to degradation of the normal system matrix condition. A posteriori interpolation of ERP to $0^{h}$ epochs gives better results. Linear interpolation was found as the most suitable for this purpose.

Table 6 shows the dependence of results on the interpolation of raw ERP series. One can see that use of linear interpolation doesn't lead to degradation of accuracy. Special tests showed that strictly daily series obtained with interpolation is more accurate than series obtained including pole coordinates rates in parameterization.

Table 6. Effect of interpolation of SLR ERP series (RMS of EOP(IAA)L - EOP(IERS)C04 after removing trend).

\begin{tabular}{lcc}
\multirow{2}{*}{ EOP } & \multicolumn{2}{c}{ Series } \\
\cline { 2 - 3 }$X, 0^{\prime \prime} 001$ & Raw & Interpolated \\
$Y, 0.001$ & 0.154 & 0.155 \\
$L O D, 0.0001 s$ & 0.171 & 0.171 \\
& 0.154 & 0.168
\end{tabular}

It should be mentioned also that producing daily solutions (independent of which method is used) provides operational solutions with a steady delay of about two days, which solves the second problem mentioned above.

Another serious problem is determination of UT from SLR (and other satellite observations). It is well known that UT1 cannot be separated from the longitude of the node of the satellite orbit during parameter solution. Three methods are being used to solve this problem.

- Fixing the longitude of the node during parameter solution (usually, during the last iteration).

- Analysis of nodal longitude series, forecasting it and using predicted values for the operational solution.

- Integrating LOD series to obtain independent free-running UT series with its possible correction for high-frequency variations from comparison with VLBI series.

Only the latter method can provide (in principle) independent results. Since that is not a subject of this paper, we will not discuss a detailed analysis of this problem. However, it is worthwhile to mention here that significant improvement of SLR UT series is also impossible without increasing the number of satellites involved in the determination of ERP.

Using the same terrestrial reference frame for all SLR solutions seems evident to achieve uniform solutions for combination. Use of the ITRF as the terrestrial reference frame by all analysis centers for their SLR solutions provides more homogeneous series for SLR combined solution.

Realization of this or alternative analysis strategy could provide more uniform, accurate operational SLR ERP series. After that, combining all submitted 
series into a final ILRS SLR product seems reasonable and useful for further use for IERS and other purposes.

We have not mentioned here the serious problem of dependence of SLR ERP results on a priori values. This is worth performing special investigations for each method used to compute ERP at various analysis centers.

\section{Conclusion}

Satellite laser ranging has made and continues to make a very valuable contribution to Earth dynamics. In particular, very valuable contributions have been made in the investigation of PM. For many years SLR was one of the main methods to determine polar motion and the main method to densify ERP series obtained with VLBI. At present five analysis centers submit operational solutions with 2-15 days delay, and about ten analysis centers contribute yearly final (up to 23 years) ERP series.

However, due to some peculiarities of this method (relatively expensive experiment, limited number of units, lack of capability of multi-satellite ranging, etc.) the quality of SLR ERP data remains the same during the last decade in spite of ranging precision improvement by a factor of a thousand from a few meters to a few millimeters since the first SLR experiments (and by factor of about 10 during the last 10-12 years). This led to decreasing weight of SLR solutions in combined ERP series.

It is evident that only a substantial increase in the number of observations and satellites involved in Earth rotation and the improvement of the distribution of observations by stations and time can lead to improvement of ERP accuracy. However, the capacity of the tracking network is practically exhausted.

On the other hand, even though GPS provides determination of ERP with impressive accuracy and delay, SLR results are very important for the combined IERS solution to improve systematic accuracy of the final IERS product. Analysis of precision of individual SLR series shows that their combination can provide combined SLR much more accurately and rapidly. To achieve the highest accuracy of combined SLR ERP product it is necessary to solve problems discussed in section 4.2 .

Realization of these opportunities by the ILRS would be very important for the investigation of Earth rotation because it would permit an independent method of the determination of PM and the velocity of the Earth's rotation.

Acknowledgments. The author is very grateful to the Scientific Organizing Committee for the invitation to the meeting and financial support of this trip.

\section{References}

Malkin, Z. 1988, presented at 1998 IERS Workshop, Potsdam, Sep 28 - Oct 2. Tapley, B. D., Schutz, B. E., Eanes, R .J., Ries, J. C., Watkins, M .M. 1993, in Contribution of Space Geodesy to Geodynamics: Earth Dynamics, D. Smith \& D. Turcotte, Washington: AGU, 147. 